\title{
Optimization design of exhaust duct system in semiconductor factory using dynamic programming method
}

\author{
Huan-Ruei Shiu*, Feng-Chu Ou, Sih-Li Chen \\ Department of Mechanical Engineering, National Taiwan University, Rooseveit Road, Taipei, Taiwan \\ Received 20 March 2002; received in revised form 16 May 2002; accepted 12 September 2002
}

\begin{abstract}
An exhaust duct system in semiconductor factory is designed using the dynamic programming method (DPM), which considers system pressure equilibrium and the least life-cycle cost to derive the duct size and fan capacity. An example of alkaline gas exhaust system is provided to understand the characteristics of DPM and to compare with the conventional duct design methods. Since DPM contains the concept of minimizing the life-cycle cost, the design results not only guarantee each path to share the same pressure, but also bear a smaller cost than other methods. The limit on duct diameter or flow velocity is added to the computation process. As a result, all the derived outcomes satisfy the requirements on the range of duct diameter or flow velocity. The differences between the design and simulation (actual operation) results under DPM are much lower than those of other methods. Thus, an exhaust duct system that best approximates the actual operation may be designed using DPM.
\end{abstract}

(C) 2003 Published by Elsevier Science Ltd.

Keywords: Exhaust duct system; Dynamic programming method

\section{Introduction}

The semiconductor industry is on a continuous course of development and improvement in both product and process at Taiwan. The number and types of special gases used in the process rise, meaning more varieties of chemical fumes are released in the air, posing greater threat to the human health and the environment. Therefore, good process exhaust duct system design becomes all the more important. The main purpose of exhaust system is to discharge waste gases generated in the process outside the plant. Exhaust volume is the first factor to be considered in the design. In addition, the air velocity in each duct section must be constrained within an acceptable range. Other problems, such as noise, vibration, pressure balance, costs and space limitations during construction have to be factored in as well. These factors are mostly inter-containing. For example, increasing design velocity or reducing duct size can lower initial costs, but in this case fan pressure needs to be increased which might require bigger fan, resulting in higher operating cost or bringing about noise or vibration problem. Thus it is always a

\footnotetext{
* Corresponding author.
}

challenge for designers to come up with an optimum exhaust system while satisfying the individual constraints.

Most conventional HVAC duct designs use the equal friction method [1] or the velocity method. These methods may be simple, but they fail to achieve pressure equilibrium. Thus, the system designed does not meet the actual operations. On-site ventilation adjustment after project completion becomes a must. In some cases, overly large fans must be installed to make up for poor design, which add to the extra costs. Although the static regain method [1] takes pressure equilibrium into consideration, it does not contain the cost concept, like other conventional design methods, and thus cannot meet the optimization requirement. T-method [2,3] is the most comprehensive and the most powerful tool applied in duct design. This method is established on a scrupulous mathematical model. It uses iteration computation and cost optimization theory, which enable the designed system to have the lowest life-cycle cost and all paths to have the same pressure loss. There is no need to waste extra time or money to attain system pressure equilibrium. However, the computation procedure of T-method is extremely complex. There are at least 20 computation steps in one iteration for only one duct section. Besides, T-method offers poor control of flow velocity or duct diameter. In cases of relatively inexpensive initial cost, the flow velocity may be too high. 


\begin{tabular}{llll}
\multicolumn{2}{l}{ Nomenclature } & & \\
$E$ & Life-cycle cost (in NT\$) & $Y$ & Annual hours of system operation $(\mathrm{h} / \mathrm{yr})$ \\
$E_{\mathrm{p}}$ & Operating energy cost for the first & $Y_{I}^{*}$ & Minimum cost (in NT\$) \\
& year (in NT\$) & $S_{\mathrm{d}}$ & Unit area cost of the ducts $\left(\mathrm{NT} \$ / \mathrm{m}^{2}\right)$ \\
$E_{\mathrm{s}}$ & Initial cost (in NT\$) & $L$ & Duct length $(\mathrm{m})$ \\
$Q_{\mathrm{fan}}$ & Fan ventilation volume $\left(\mathrm{m}^{3} / \mathrm{s}\right)$ & $C$ & Local loss coefficient \\
$P_{\mathrm{fan}}$ & Fan static pressure $(\mathrm{Pa})$ & $D$ & Diameter of circular duct $(\mathrm{m})$ \\
$\eta_{\mathrm{f}}$ & Fan efficiency $(\%)$ & $H$ & Height of square duct $(\mathrm{m})$ \\
$\eta_{\mathrm{e}}$ & Efficiency of the drive motor $(\%)$ & $W$ & Width of square duct $(\mathrm{m})$ \\
$E_{\mathrm{c}}$ & Unit price of electricity $(\mathrm{NT} \$ / \mathrm{kW}-\mathrm{h})$ & &
\end{tabular}

In contrast, for cases of relatively inexpensive energy cost, the duct size may be too big. Nevertheless, in actual duct design, considerations of the factors of space and noise often require a limit on the duct diameter or flow velocity during certain sections. When there are too many limitations, it is difficult to obtain the satisfactory and stable optimal solution from T-method, as discussed by Mathews and Claassen [4]. Thus, it is necessary to search for a design method that contains a simple computation procedure, considers the least life-cycle cost and pressure equilibrium under certain limits on space or flow velocity.

Dynamic programming method (DPM) is a type of mathematical technique first developed by Richard Bellman [5]. It is an optimization method extremely suitable for use in analyzing problems with a complex and multiple stage decision-making procedure and searching for the best strategy. Bellman believes that the optimal decision should possess the following characteristic: "Regardless of the initial conditions and initial decisions, the future condition and decision resulted from these initial conditions and decisions must be able to produce the best solution for the problem". In other words, if the current states and the devised decisions are known conditions, the best policy they produce in the future must be independent of the previous policy. Thus, the problems to be solved using dynamic programming are mostly decision-making problems with multiple stages that can be divided. In particular, dynamic programming is very suitable for solving the optimization problem whose objective function cannot be differentiated. The optimization design of a duct system happens to conform to such an application.

Problems that can be solved by dynamic programming must possess the following four characteristics [6]:

1. The problem must be able to be divided into several stages, in which a decision needs to be made in each stage. For instance, in solving the optimization problem of a duct system, the problem is divided into stages according to the duct nodes.

2. Each stage has a state vector, which consists of a set of state variables that describe the system conditions such as the pressure value of the duct.
3. A certain decision vector in each stage must be able to transform a certain state vector in that stage into a certain state vector in the next stage. The decision vector consists of a set of decision variables, which represent the decisions made in relation to the system during a certain stage. The effect of these decisions on the system can be expressed in an appropriate measurement. Usually, this effect is quantified through the objective function.

4. As far as each state of any one stage is concerned, the optimal decision made is unrelated to the decision made during the previous stage. This means that the state variables of the current stage already contain all impact on the system resulting from the decisions made during the previous stage.

To sum up the above, the optimization design problem of the duct system satisfies all of the above characteristics, and is thus very suitable for using DPM for solution. The objective of this paper is to apply the method in order to develop an optimal duct design method that better meets practical applications.

\section{Objective function}

The objective of an optimal HVAC duct system design is to search for the pipeline combination with the lowest total system pressure loss in order to optimize the total cost, given the known conditions of ventilation volume at each ventilation exit. Thus, it is necessary to use the life-cycle cost of the system as the objective function of optimization. The life-cycle cost can then be minimized through the selection of the optimal duct diameter and optimal fan pressure.

The life-cycle cost of a duct system includes the initial cost and operating energy cost. The initial cost includes the duct price and installation cost, while the operating energy cost includes energy charge and energy demand. The optimization method can derive the fan static pressure that minimizes costs. Since many of the costs mentioned above are constants and unrelated to optimization, only the initial cost and energy cost need to be included in the objective 
function, which can be expressed as in Eq. (1):

$E=E_{\mathrm{p}}(\mathrm{PWEF})+E_{\mathrm{s}}$,

where $E$ is the life-cycle cost (in NT\$), $E_{\mathrm{p}}$ the operating energy cost for the first year (in NT\$), and PWEF is the present worth escalation factor, as shown in Eq. (2).

$P W E F=\frac{\left(\frac{1+A E R}{1+A I R}\right)^{\mathrm{a}}-1}{1-\left(\frac{1+A I R}{1+A E R}\right)}$

where $A E R$ is the annual escalation rate; $A I R$ the annual interest rate; and a the amortization period.

The system's initial cost is actually the cost of power consumption. Since the primary power consumption comes from the fan, thus

$E_{p}=\frac{Q_{\mathrm{fan}} P_{\mathrm{fan}}}{\eta_{\mathrm{f}} \eta_{\mathrm{e}}} E_{\mathrm{c}} Y$

where $Q_{\text {fan }}$ is the fan ventilation volume $\left(\mathrm{m}^{3} / \mathrm{s}\right), P_{\text {fan }}$ the fan static pressure $(\mathrm{Pa}), \eta_{\mathrm{f}}$ the fan efficiency $(\%), \eta_{\mathrm{e}}$ the efficiency of the drive motor $(\%), E_{\mathrm{c}}$ the unit price of electricity (NT\$/kW-h), and $Y$ the annual hours of system operation $(\mathrm{h} / \mathrm{yr})$.

The system's initial cost can be calculated by multiplying the cost per unit area of the ducts by the total duct area. This unit area cost should include material cost, delivery cost and wages for installation and construction. The cost of all accessories should also be included.

For duct sections with circular cross sections

$E_{\mathrm{s}}=S_{\mathrm{d}}(\pi D L)$

For duct sections with square cross sections

$E_{\mathrm{s}}=2 S_{\mathrm{d}}(H+W) L$

where $S_{\mathrm{d}}$ is the unit area cost of the ducts $\left(\mathrm{NT} \$ / \mathrm{m}^{2}\right), L$ the duct length (m), $D$ the diameter of circular duct (m), $H$ the height of square duct (m), and $W$ the width of square duct (m).

Incorporate Eqs. (2)-(4) into Eq. (1) to obtain the life-cycle costs of circular ducts and square ducts.

For circular ducts

$E=\left(\frac{Q_{\mathrm{fan}} P_{\mathrm{fan}}}{\eta_{\mathrm{f}} \eta_{\mathrm{e}}} E_{\mathrm{c}} Y\right) \mathrm{PWEF}+S_{\mathrm{d}}(\pi D L)$.

For square ducts

$E=\left(\frac{Q_{\text {fan }} P_{\text {fan }}}{\eta_{\mathrm{f}} \eta_{\mathrm{e}}} E_{\mathrm{c}} Y\right) \mathrm{PWEF}+2 S_{\mathrm{d}}(H+W) L$.

In this paper, all ducts are assumed to be circular. Hence, the objective function will use Eq. (5a) as the basis for calculation.

Among the settings of parameters related to costs, fan efficiency is set at $80 \%$ and motor efficiency at $80 \%$. The unit area price of duct is based on NT $\$ 500 / \mathrm{m}^{2}$, and, sum of the costs of corner materials, frames and the miscellaneous expenses of delivery cost and wages set at $30 \%$, or
NT $\$ 650 / \mathrm{m}^{2}$. As for the calculation of PWEF, the system is assumed to last for a period of 10 years. Given interest rate and escalation for energy cost at $6 \%$ and $3 \%$, respectively, the PWEF value is derived at 8.568 .

\section{Dynamic programming method}

Dynamic programming method (DPM) can be divided into the forward DP approach and backward DP approach depending on the direction of operation. The forward approach begins the calculation from the first stage to the final stage and then retraces from the final stage to the front to determine the optimal decision. The backward approach begins from the final stage to the first and then follows the sequence from the first stage on to determine the optimal decision. Regardless of the approach, the main purpose is to search for the optimal path in order to achieve lowest costs.

The backward approach is adopted in this paper, as shown in Fig. 1. The optimal path from Stage I +1 to Stage I is determined through recursion. The recursion equation is written as Eq. (6):

$Y_{I}^{*}(s)=\operatorname{Min}_{x i}\left[Y_{I}\left(s, x_{I}\right)+Y_{I+1}^{*}\left(x_{I}\right)\right]$.

As far as general dynamic programming methods are concerned, given an $S$ number of conditions in each stage, then there are a total of $S^{n-1}$ types of possible paths after $(N-1)$ stages. If the backward approach of DPM is adopted, it takes only $(n-2) S^{2}+S$ times of operations to complete the computation. The efficiency of deriving the solution is very significant. Unlike linear programming, there are no standard problem solution methods or comprehensive software packages available for dynamic programming. Thus, it often becomes necessary to develop a computer program in order to solve certain problem. Thus, it is not as convenient to develop programs for dynamic programming as that for linear programming. However, dynamic programming is not constrained by the numerous constraints linear programming is subject to during standardization. Thus, its objective function and constraints are not required to be linear. It is not necessary to oversimplify the actual system either, which enables the design to maintain the system characteristics.

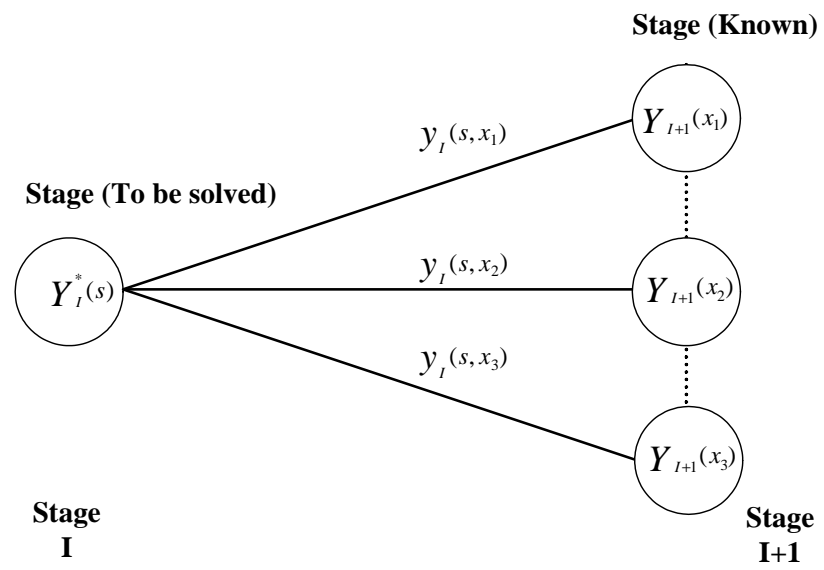

Fig. 1. Model of backward DPM. 


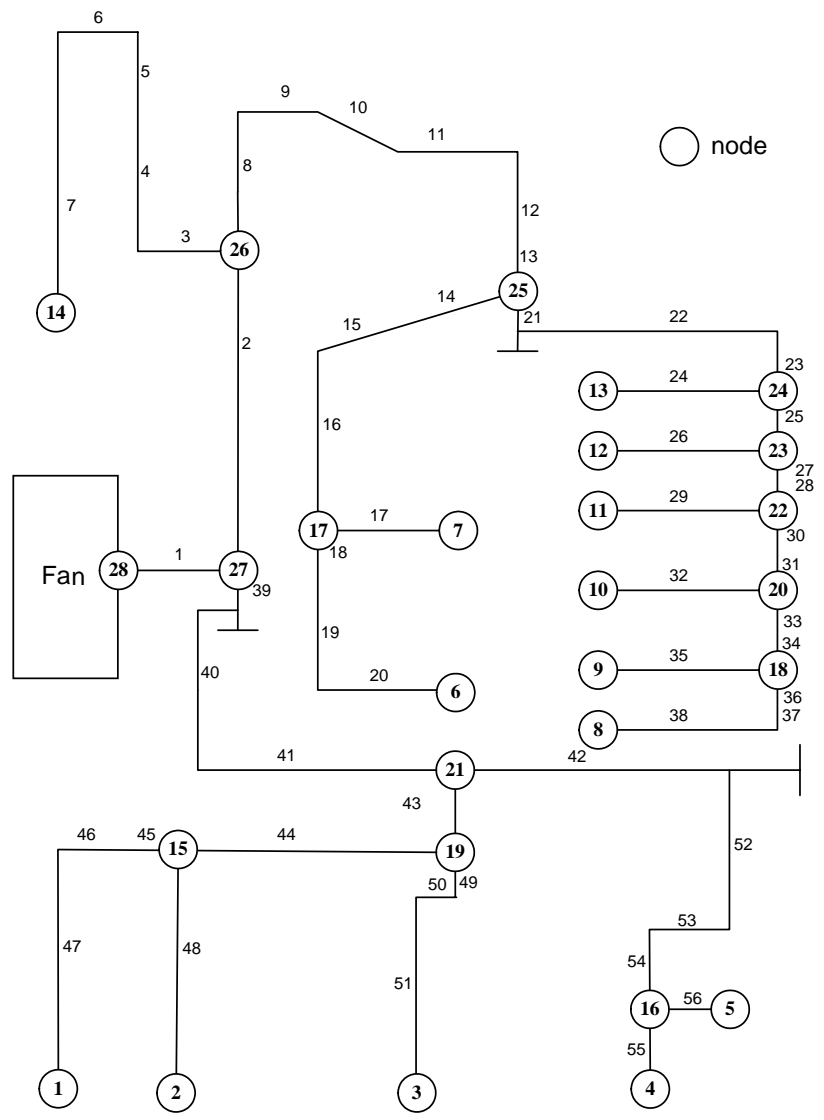

Fig. 2. Nodes configuration under DPM.

DPM is used in the design and analysis of alkaline gas exhaust duct system of a semiconductor plant. Fig. 2 depicts the exhaust system configuration. The length of each duct section in the system depends on equipment location and architectural structure. In Fig. 2, duct sections are numbered by different flow rate, duct size and shape of cross section, and divided into 56 sections and 14 paths. The flow rate and section length are known conditions, as shown in Table 1. In consideration of control, connection and safety, the duct system is installed with relevant fittings [1]. The design points are jointly determined by constraints, plant requirements, and exhaust flow rate, the paramount of which are safety velocity and total exhaust volume. As shown in Table 2, the maximum and minimum of safety velocity are determined by parameters, including type of exhaust, humidity, vibration and noise as suggested in manufacturer or reference handbook [1].

The first step in the calculation is to set nodes based on the principle where "nodes are required at duct divergence (at confluence or distribution point) and air outlet (or return)". Fig. 2 presents the configuration of system nodes. What follows is to stratify the duct system: set the total pressure on path terminal at 0 , then calculate from level 1 toward the last level, and calculate all acceptable total pressure, duct size and air velocity on every node from level 1 to the last level. Substitute the total pressure and total sec-
Table 1

Sectional length and flow rate

\begin{tabular}{|c|c|c|}
\hline Duct no. & Flow rate $\left(\mathrm{m}^{3} / \mathrm{s}\right)$ & Section length $(\mathrm{m})$ \\
\hline 1 & 10.620 & 1.000 \\
\hline 2 & 8.280 & 38.000 \\
\hline 3 & 0.370 & 3.800 \\
\hline 4 & 0.370 & 3.000 \\
\hline 5 & 0.370 & 1.500 \\
\hline 6 & 0.370 & 9.600 \\
\hline 7 & 0.370 & 12.600 \\
\hline 8 & 7.910 & 0.500 \\
\hline 9 & 7.910 & 2.500 \\
\hline 10 & 7.910 & 4.500 \\
\hline 11 & 7.910 & 10.500 \\
\hline 12 & 7.910 & 14.700 \\
\hline 13 & 7.910 & 4.000 \\
\hline 14 & 2.110 & 0.500 \\
\hline 15 & 2.110 & 6.400 \\
\hline 16 & 2.110 & 20.400 \\
\hline 17 & 1.060 & 23.000 \\
\hline 18 & 1.050 & 2.000 \\
\hline 19 & 1.050 & 47.600 \\
\hline 20 & 1.050 & 20.200 \\
\hline 21 & 5.800 & 0.500 \\
\hline 22 & 5.800 & 57.000 \\
\hline 23 & 5.800 & 6.000 \\
\hline 24 & 0.530 & 22.000 \\
\hline 25 & 5.270 & 16.000 \\
\hline 26 & 0.500 & 22.000 \\
\hline 27 & 4.770 & 1.000 \\
\hline 28 & 4.770 & 5.400 \\
\hline 29 & 1.720 & 21.400 \\
\hline 30 & 3.050 & 1.000 \\
\hline 31 & 3.050 & 13.400 \\
\hline 32 & 1.720 & 21.400 \\
\hline 33 & 1.330 & 1.000 \\
\hline 34 & 1.330 & 6.000 \\
\hline 35 & 0.720 & 18.000 \\
\hline 36 & 0.610 & 1.000 \\
\hline 37 & 0.610 & 14.400 \\
\hline 38 & 0.610 & 22.400 \\
\hline 39 & 2.340 & 1.000 \\
\hline 40 & 2.340 & 3.200 \\
\hline 41 & 2.340 & 2.000 \\
\hline 42 & 0.820 & 2.000 \\
\hline 43 & 1.520 & 15.000 \\
\hline 44 & 1.170 & 3.000 \\
\hline 45 & 0.700 & 1.000 \\
\hline 46 & 0.700 & 7.000 \\
\hline 47 & 0.700 & 12.000 \\
\hline 48 & 0.470 & 12.000 \\
\hline 49 & 0.330 & 17.500 \\
\hline 50 & 0.330 & 8.600 \\
\hline 51 & 0.330 & 10.400 \\
\hline 52 & 0.820 & 21.500 \\
\hline 53 & 0.820 & 15.000 \\
\hline 54 & 0.820 & 24.000 \\
\hline 55 & 0.560 & 5.000 \\
\hline 56 & 0.260 & 10.200 \\
\hline
\end{tabular}

tional area into objective function to obtain optimum value after comparison. In level layout, level 1 must be related to air outlet (or air return), while the last level is the fan side. The whole calculation process is carried out backward from 
Table 2

Design point of an alkaline gas exhaust system

\begin{tabular}{ll}
\hline Constraint & Parameter value \\
\hline Min. safety velocity & $5 \mathrm{~m} / \mathrm{s}$ \\
Max. safety velocity & $15 \mathrm{~m} / \mathrm{s}$ \\
Design velocity & $7.5 \mathrm{~m} / \mathrm{s}$ \\
Total air volume required & $10.70 \mathrm{~m}^{3} / \mathrm{s}$ \\
\hline
\end{tabular}

air outlet toward the fan. The principle of stratification is: "required data for nodes in level $n+1$ must include known results of level $n$ ". Nine levels are obtained from the stratification: Level 1 contains nodes 15, 16, 17 and 18, Level 2 contains nodes 19 and 20, Level 3 contains nodes 21 and 22, Level 4 contains nodes 23, Level 5 contains node 24, Level 6 contains 25, Level 7 contains node 26, Level 8 contains node 27, and Level 9 contains node 28. Given that nodes 1 through 14 are connected with the atmosphere, their total pressure values are 0, i.e. $P_{\mathrm{t}_{1}}=P_{\mathrm{t}_{2}}=P_{\mathrm{t}_{3}}=\cdots P_{\mathrm{t}_{14}}=0$.

The design for Level 1 with nodes $15,16,17$ and 18 is carried out first. Since each node is independent of each other, calculation may start with any of the nodes. This study first considers the total pressure on node 15 , which has a range of 1-600 $\mathrm{Pa}$ with an increment interval of $1 \mathrm{~Pa}$. The maximum total pressure value can be adjusted arbitrarily based on the size of duct. The maximum total pressure for this duct system is set at $600 \mathrm{~Pa}$. When the pressure of node 15 is $P_{15}=P_{15}^{(1)}=1 \mathrm{~Pa}$, the total pressure drop from node 15 to node 1 and node 2 is, respectively,

$\Delta P_{15-1}^{(1)}=P_{15}^{(1)}-P_{1}$,

$\Delta P_{15-2}^{(1)}=P_{15}^{(1)}-P_{2}$.

Given that $P_{1}=P_{2}=0$, the equations above may be re-written as

$\Delta P_{15-1}^{(1)}=P_{15}^{(1)}$,

$\Delta P_{15-2}^{(1)}=P_{15}^{(1)}$.

The duct diameter may be obtained from the total pressure drop using the following equations:

$\Delta P_{l}=\left(\frac{f L}{D}+C\right) \frac{\rho V^{2}}{2}$,

$f=0.11\left(\frac{\varepsilon}{D}+\frac{68}{R e}\right)^{0.25}$

$V^{2}=\frac{16 Q^{2}}{\pi^{2} D^{4}}$

$R e=\frac{V D}{v}$.
Substitute Eqs. (8b), (8c) and (8d) into (8a) to obtain (8e),

$$
\begin{aligned}
\Delta P_{l}= & {\left[0.11\left(\frac{\varepsilon}{D}+\frac{17 \pi D v}{Q}\right)^{0.25} \frac{L}{D}+C\right] } \\
& \left(\frac{\rho}{2} \frac{16 Q^{2}}{\pi^{2} D^{4}}\right) .
\end{aligned}
$$

From (8e), solutions for node 15 at Level 1 may be expressed as follows:

$$
\begin{aligned}
& \Delta P_{15-1}= {\left[0.11\left(\frac{\varepsilon}{D_{15-1}}+\frac{17 \pi D_{15-1} v}{Q_{15-1}}\right)^{0.25}\right.} \\
&\left.\times \frac{L_{15-1}}{D_{15-1}}+C_{15-1}\right]\left(\frac{\rho}{2} \frac{16 Q_{15-1}^{2}}{\pi^{2} D_{15-1}^{4}}\right), \\
& \Delta P_{15-2}= {\left[0.11\left(\frac{\varepsilon}{D_{15-2}}+\frac{17 \pi D_{15-2} v}{Q_{15-2}}\right)^{0.25}\right.} \\
&\left.\times \frac{L_{15-2}}{D_{15-2}}+C_{15-2}\right]\left(\frac{\rho}{2} \frac{16 Q_{15-2}^{2}}{\pi^{2} D_{15-2}^{4}}\right), \\
& D_{\min } \leqslant D_{15-1} \leqslant D_{\max }, \\
& D_{\min } \leqslant D_{15-2} \leqslant D_{\max } \\
& V_{\min } \leqslant V_{15-1} \leqslant V_{\max }, \\
& V_{\min } \leqslant V_{15-2} \leqslant V_{\max } .
\end{aligned}
$$

From Eq. (9), the corresponding duct size may be derived if total pressure drop is known. But the said equation does not produce duct diameter directly. A numerical method needs to be applied and Newton method is used in this paper. If the resulting diameter or velocity cannot satisfy the constraints set in (10a)-(10d), the pressure value will be rejected. The so-called unacceptable duct are sizes that exceed the space allowed, which is defined at $1.5 \mathrm{~m}$ in this paper.

After obtaining $D_{15-1}^{(1)}$ and $D_{15-2}^{(1)}$, the initial costs of these two duct sections $E s_{15-1}^{(1)}$ and $E s_{15-2}^{(1)}$ may be computed using Eq. (5a). Then calculate the corresponding duct size, velocity and initial cost under the rest of total pressure by the increment of $1 \mathrm{~Pa}$. These acceptable values are stored in the computer in arrays, as shown in Table $3 . E s_{15}^{(1)}$, the farthest right column of Table 3, represents the total sectional area (the sum of surface area of duct sections 45, 46, 47 and 48) connected to node 15 under $P_{15}^{(1)}$, and so on. Given that node 15 is connected to the outlet at the end of the ductwork, $E s_{15-1}^{(1)}$ may also be taken as the minimum total sectional area under $P_{15}^{(1)}$ after node 15 , and so on. The same operation may be applied to the calculations for nodes 16,17 and 18 .

The same design method just described is applied to the next level, up to Level 9 where node 28 is the fan side. Thus the total pressure on node 28 is the total pressure the fan 
Table 3

Pressure value and initial cost of designed nodes at Level 1

\begin{tabular}{|c|c|c|c|c|c|}
\hline $\begin{array}{l}\text { Node } 15 \\
P_{15}^{(1)} \\
P_{15}^{(2)}\end{array}$ & $\begin{array}{l}D_{15-1}^{(1)} \\
D_{15-1}^{(2)}\end{array}$ & $\begin{array}{l}V_{15-1}^{(1)} \\
V_{15-1}^{(2)}\end{array}$ & $\begin{array}{l}D_{15-2}^{(1)} \\
D_{15-2}^{(2)}\end{array}$ & $\begin{array}{l}V_{15-2}^{(1)} \\
V_{15-2}^{(2)}\end{array}$ & $\begin{array}{l}E s_{15-1}^{(1)}+E s_{15-2}^{(1)}=E s_{15}^{(1)} \\
E s_{15-1}^{(2)}+E s_{15-2}^{(2)}=E s_{15}^{(2)}\end{array}$ \\
\hline$P_{15}^{(S)}$ & $D_{15-1}^{(S)}$ & $\begin{array}{l}\cdots \\
V_{15-1}^{(S)}\end{array}$ & $\begin{array}{l}\cdots \\
D_{15-2}^{(S)}\end{array}$ & $\begin{array}{l}\cdots \\
V_{15-2}^{(S)}\end{array}$ & $E s_{15-1}^{(S)}+E s_{15-2}^{(S)}=E s_{15}^{(S)}$ \\
\hline $\begin{array}{l}\text { Node } 16 \\
P_{16}^{(1)} \\
P_{16}^{(2)}\end{array}$ & $\begin{array}{l}D_{16-4}^{(1)} \\
D_{16-4}^{(2)}\end{array}$ & $\begin{array}{l}V_{16-4}^{(1)} \\
V_{16-4}^{(2)}\end{array}$ & $\begin{array}{l}D_{16-5}^{(1)} \\
D_{16-5}^{(2)}\end{array}$ & $\begin{array}{l}V_{16-5}^{(1)} \\
V_{16-5}^{(2)}\end{array}$ & $\begin{array}{l}E s_{16-4}^{(1)}+E s_{16-5}^{(1)}=E s_{16}^{(1)} \\
E s_{16-4}^{(2)}+E s_{16-5}^{(2)}=E s_{16}^{(2)}\end{array}$ \\
\hline $\begin{array}{l}\cdots \\
P_{16}^{(S)}\end{array}$ & $\ddot{D}_{16-4}^{(S)}$ & $\begin{array}{l}\cdots \\
V_{16-4}^{(S)}\end{array}$ & $\begin{array}{l}\cdots \\
D_{16-5}^{(S)}\end{array}$ & $\begin{array}{l}\cdots \\
V_{16-5}^{(S)}\end{array}$ & $E s_{16-4}^{(S)}+E s_{16-5}^{(S)}=E s_{16}^{(S)}$ \\
\hline $\begin{array}{l}\text { Node } 17 \\
P_{17}^{(1)} \\
P_{17}^{(2)}\end{array}$ & $\begin{array}{l}D_{17-7}^{(1)} \\
D_{17-7}^{(2)}\end{array}$ & $\begin{array}{l}V_{17-7}^{(1)} \\
V_{17-7}^{(2)}\end{array}$ & $\begin{array}{l}D_{17-7}^{(1)} \\
D_{17-6}^{(2)}\end{array}$ & $\begin{array}{l}V_{17-6}^{(1)} \\
V_{17-6}^{(2)}\end{array}$ & $\begin{array}{l}E s_{17-7}^{(1)}+E s_{17-6}^{(1)}=E s_{17}^{(1)} \\
E s_{17-7}^{(2)}+E s_{17-6}^{(2)}=E s_{17}^{(2)}\end{array}$ \\
\hline $\begin{array}{l}\cdots \\
P_{17}^{(S)}\end{array}$ & $\bar{D}_{17-7}^{(S)}$ & $\begin{array}{l}\cdots \\
V_{17-7}^{(S)}\end{array}$ & $\begin{array}{l}\cdots \\
D_{17-6}^{(S)}\end{array}$ & $\begin{array}{l}\cdots \\
V_{17-6}^{(S)}\end{array}$ & $E s_{17-7}^{(S)}+E s_{17-6}^{(S)}=E s_{17}^{(S)}$ \\
\hline $\begin{array}{l}\text { Node } 18 \\
P_{18}^{(1)} \\
P_{18}^{(2)}\end{array}$ & $\begin{array}{l}D_{18-8}^{(1)} \\
D_{18-8}^{(2)}\end{array}$ & $\begin{array}{l}V_{18-8}^{(1)} \\
V_{18-8}^{(2)}\end{array}$ & $\begin{array}{l}D_{18-9}^{(1)} \\
D_{18-9}^{(2)}\end{array}$ & $\begin{array}{l}V_{18-9}^{(1)} \\
V_{18-9}^{(2)}\end{array}$ & $\begin{array}{l}E s_{18-8}^{(1)}+E s_{18-9}^{(1)}=E s_{18}^{(1)} \\
E s_{18-8}^{(2)}+E s_{18-9}^{(2)}=E s_{18}^{(2)}\end{array}$ \\
\hline$P_{18}^{(S)}$ & $D_{18-8}^{(S)}$ & $\begin{array}{l}\cdots \\
V_{18-8}^{(S)}\end{array}$ & $\begin{array}{l}\cdots \\
D_{18-9}^{(S)}\end{array}$ & $\begin{array}{l}\cdots \\
V_{18-9}^{(S)}\end{array}$ & $E s_{18-8}^{(S)}+E s_{18-9}^{(S)}=E s_{18}^{(S)}$ \\
\hline
\end{tabular}

should supply. The equation for the solutions on this level is expressed below:

$$
\begin{aligned}
\Delta P_{28-27}= & {\left[0.11\left(\frac{\varepsilon}{D_{28-27}}+\frac{17 \pi D_{28-27} v}{Q_{28-27}}\right)^{0.25} \frac{L_{28-27}}{D_{28-27}}\right.} \\
& \left.+C_{28-27}\right]\left(\frac{\rho}{2} \frac{16 Q_{28-27}^{2}}{\pi^{2} D_{28-27}^{4}}\right),
\end{aligned}
$$

$D_{\min } \leqslant D_{28-27} \leqslant D_{\max }$,

$V_{\min } \leqslant V_{28-27} \leqslant V_{\max }$.

As described, there might be a number of options of $P_{27}$ for $P_{28}$. Thus the options must be compared to obtain $P_{27}$ with the smallest sectional area. The resulting parameters are listed in Table 3, in which $P_{28,27}^{(1)}$ represents the total pressure of node 27 corresponding to $P_{28}^{(1)}$, whereas $E s_{28}^{(1)}$ in the farthest right column represents the minimum sectional area of the entire ductwork. That is when the total pressure on node 28 is $P_{28}^{(1)}$ and the total pressure on node 27 is $P_{28,27}^{(1)}$, the minimum area of the entire ductwork is $E s_{28}^{(1)}$.

After calculating all acceptable total pressure values $P_{28}$ on node 28 and its corresponding total initial costs $E s_{28}$, we will proceed with the comparison of minimum life-cycle cost. Given that $P_{28}$ represents the fan pressure required which is related to initial cost, the minimum value of objective function may be selected by substituting $E s_{28}$ and $P_{28}$ into objective function and by comparison. The minimum operating and initial cost in the life cycle are the optimum design values for the duct system.

\section{Results and discussion}

The main purpose of an exhaust system is to discharge waste gases generated in the process outside the plant through the ductwork. If the air velocity is too low, solid granules may not be discharged, but instead, build up inside the ductwork and pose potential hazard. Thus the air velocity inside the ductwork must be maintained above an allowed lower limit. On the other hand, air velocity that is too high might cause noise or vibration problem, thus the designed velocity may not exceed an allowed upper limit. From Fig. 3 one can see that the velocity method set the velocity in each section as equal, mainly because velocity is kept at a constant under known design points. Under static regain method, the designed system velocity is relatively low and some sections have velocity below the minimum allowed. This is because under static regain method, "At the nodes of the duct system, the dynamic pressure is lowered in exchange of rising static pressure and the static regain is used to offset the loss of static pressure drop from friction in subsequent section after the node". Thus although the decrease of dynamic pressure exchanged for the rise of static pressure, the air velocity is reduced as a result. Under equal friction method, the average velocity is relatively high. The system velocity designed by DPM also tends to be high, but within allowable range. This is because its total pressure loss tended to be high. The tendency of rise in velocity is also observed in Eq. (8a), mainly because total pressure loss is directly proportional to the square of velocity. The tendency of high total pressure is also seen in Fig. 4. 


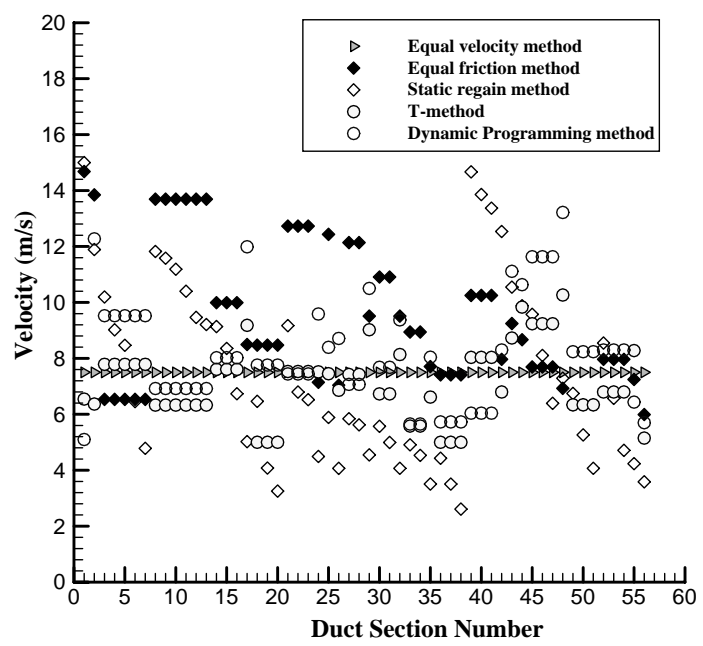

Fig. 3. Comparison of design point velocity under different methods.

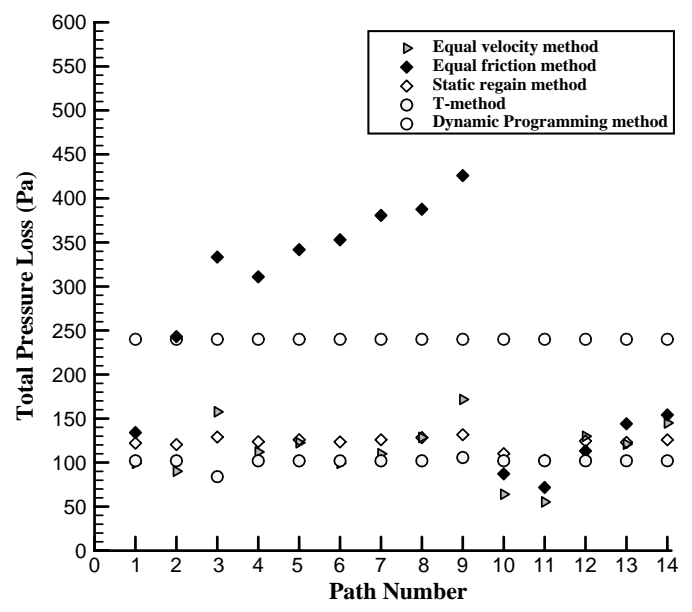

Fig. 4. Comparison of total pressure loss.

In actual operation, the total pressure of the exhaust system will adjust automatically to attain a balance, that is, the total pressure drop in each path becomes equal. If pressure balance is not achieved in the design stage, the velocity and flow in each duct section will not meet the design needs. That means more time and money need to be spent on air flow adjustment after the system is constructed. In other words, the closer the designed pressure loss of each path to the other, the more approximate the system will be to the real scenario. From Fig. 4 one can see that the system pressure values designed by dynamic programming are more equal. This is because pressure balance is a basic constraint under this method. T-method also has the constraint of pressure balance, but it will be sacrificed when other constraints, such as duct size or velocity are included. In this case, pressure imbalance is resulted in the design using T-method due to the constraint of velocity range. In the design using static regain method, the static regain factor is set as 1.0 , meaning the static pressures at upstream and downstream of the

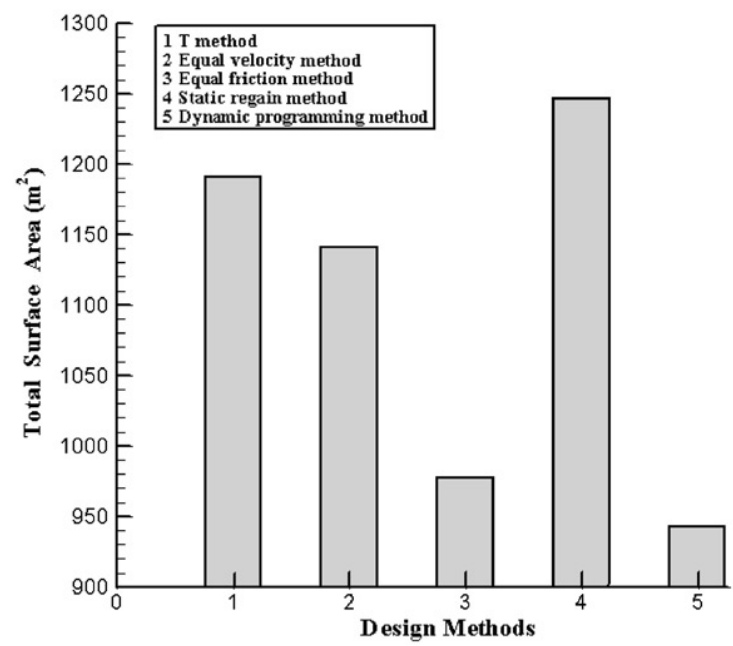

Fig. 5. Comparison of total surface area under different methods.

distributing duct are equal. Thus a fairly pressure balanced system is derived. It is also shown in Fig. 4 that the pressure imbalance rate under velocity method is rather high, next only to equal friction method. Equal friction method produces a design with the worst pressure balance. This is because the basic assumption of the method is "all sections of the system have equal unit length pressure loss". Thus it is more suitable for the design of a symmetrical system. But for an asymmetrical system as the case here, a system with disparate system pressure is resulted using equal friction method.

The total surface area of the exhaust system represents its initial cost. To cut down initial cost, duct size needs to be reduced. But reduced duct size will result in elevation of velocity and pressures, and high velocity will produce noise and vibration problems. If the pressure drop is too high, a larger fan will be required. Thus the determination of duct size, velocity and pressure drop is a big challenge for the designer. Duct size is also related to total surface area of the entire ductwork. Fig. 5 shows that the design under DPM produces the smallest total surface area, meaning its initial cost would be the smallest in comparison with other methods, followed by equal friction method. Static regain method results in the highest initial cost, because it has bigger duct size and requires lower fan pressure.

Testing the performance of the designed system in actual operation may be carried out by means of experiment or computer simulation. To save cost and facilitate change of design, computer-aided simulation is employed for testing purpose. System simulation is modeled after a method proposed by Tsal et al. [7]. The said method may apply to simulation of a designed duct system where the flow rate and velocity in each duct section during system operation are computed with known duct size conditions and data of fittings. Fig. 6 shows that velocities derived from DPM and T-method mostly fall within the originally designed limit. This is because both methods factored in velocity 


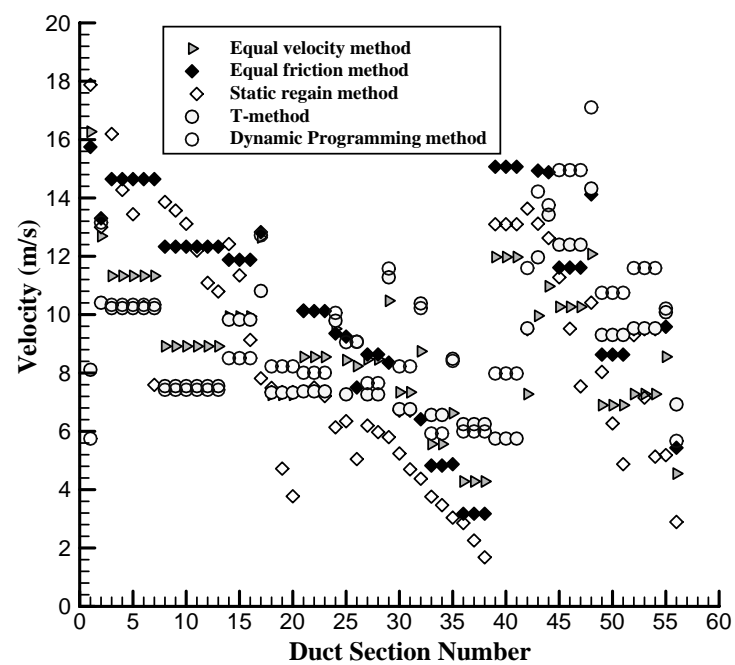

Fig. 6. Comparison of simulated sectional velocity under different methods.

Table 4

Pressure value and initial cost of designed nodes at Level 9

\begin{tabular}{lllll}
\hline Node 28 & & & & \\
$P_{28}^{(1)}$ & $D_{28-27}^{(1)}$ & $V_{28-27}^{(1)}$ & $P_{28,27}^{(1)}$ & $E s_{28-27}^{(1)}+E s_{27}^{(\min )}=E s_{28}^{(1)}$ \\
$P_{28}^{(2)}$ & $D_{28-27}^{(2)}$ & $V_{28-27}^{(2)}$ & $P_{28,27}^{(2)}$ & $E s_{28-27}^{(2)}+E s_{27}^{(\min )}=E s_{28}^{(2)}$ \\
$\cdots$ & $\cdots$ & $\cdots$ & $\cdots$ & $\cdots$ \\
$P_{28}^{(S)}$ & $D_{28-27}^{(S)}$ & $V_{28-27}^{(S)}$ & $P_{28,27}^{(S)}$ & $E s_{28-27}^{(3)}+E s_{27}^{(\min )}=E s_{28}^{(3)}$ \\
\hline
\end{tabular}

limitations. Hence their simulation results are consistent with the design values. Other conventional design methods (velocity, equal friction and static regain) also set velocity limit, but the limitation is unidirectional (set the velocity under certain value), instead of being in a specific range. Thus Fig. 6 shows that the distribution of velocity values obtained from these methods is not within the set range. Static regain method produces the widest distribution range and relatively low system velocity (Table 4 ).

Table 5 shows that flow errors under the three conventional methods were relatively big; velocity method had error up to $68.49 \%$, and equal friction method had error up to $124.86 \%$, followed by $104.04 \%$. The biggest error under static regain method is $58.92 \%$, and that under T-method is $81.92 \%$. DPM produces smallest errors overall with the biggest being $25.71 \%$. It is also found that the three conventional design methods have similar error tendency, meaning that when one of the methods have bigger error at a certain return outlet, the same situation occurs with the other two methods. This is because these three methods did not set strict limits on parameters such as duct size, velocity and air flow since optimal life-cycle cost was not taken into consideration. Thus their flow control is far inferior to that of T-method and DPM, which shows less flow error. DPM produces the smallest error of all, because it can find the optimal design while satisfying all constraints. T-method has the same objective functions as dynamic programming. But

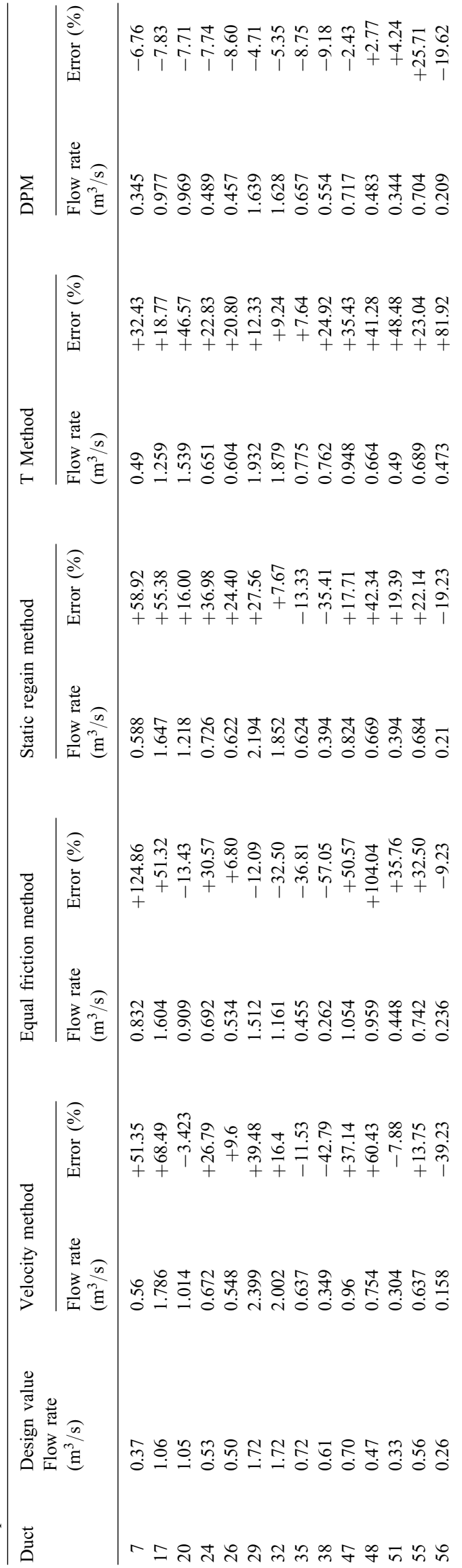


the total pressure drop obtained from this method will not be balanced with the inclusion of other constraints (such as duct size or air velocity) and cannot meet the requirement of convergence, meaning the design is not a real optimal design. DPM shows small errors in paths other than path 13. This is because the pressure loss coefficient of some sections on path 13 does not have the correct setting. Overall, DPM produces the smallest air flow error, while system designed under equal friction method shows largest error.

Figs. 7-9 compare the initial cost, energy cost and life-cycle cost of the system. Fig. 7 shows that initial cost was the lowest with DPM and highest with static regain method, because the latter designs the largest duct size and the former produces the smallest duct size. Fig. 8 shows that the energy cost was the lowest with DPM and highest with equal friction method, because the former has the smallest value from operating point pressure multiplied by air flow and the latter provides the largest such value. Life-cycle cost is the sum of initial cost and operating cost. Fig. 9 indicates that both DPM and T-method could achieve minimization of life-cycle cost with the former producing the smallest life-cycle cost. Aside from the life-cycle cost, some systems with pressure imbalance would require damper or other balancing devices for pressure adjustment, which also adds to the cost. In fact, except for DPM, other methods of design would face the problem of pressure imbalance under the constraints and incur additional cost for flow rate adjustment.

If we further compare the results between DPM and the T-method, we can learn that T-method produces the smallest fan static pressure, while DPM generates the smallest total duct area. From the perspective of current engineering design and application, designers often base their design on the maximum air-conditioning load. However, inverter is often adopted in conjunction with changes in load in order to reduce fan output and lower the power cost. Therefore, even if the fan static pressure designed by DPM is slightly higher, this weakness can be improved in realistic operation. In contrast, the total duct area affects the initial cost, and cannot be adjusted in any way in the operation afterwards. Besides, among the total cost of a duct system, the initial cost accounts for $80 \%$ of the life-cycle cost. Thus, only the reduction of the total duct area can truly lower the cost of a duct system. DPM can design a duct system with the least total duct area. Together with a simple computation process, DPM does offer exceptional design advantages.

\section{Conclusions}

Since DPM and the T-method contain the concept of minimal life-cycle cost in their design, their design results are able to achieve lower costs than other design methods. In addition, constraints of the duct diameter and flow velocity can be added to the computation process of DPM. When the flow velocity or duct diameter values exceed the designated allowable range, such values are eliminated. Thus, all the

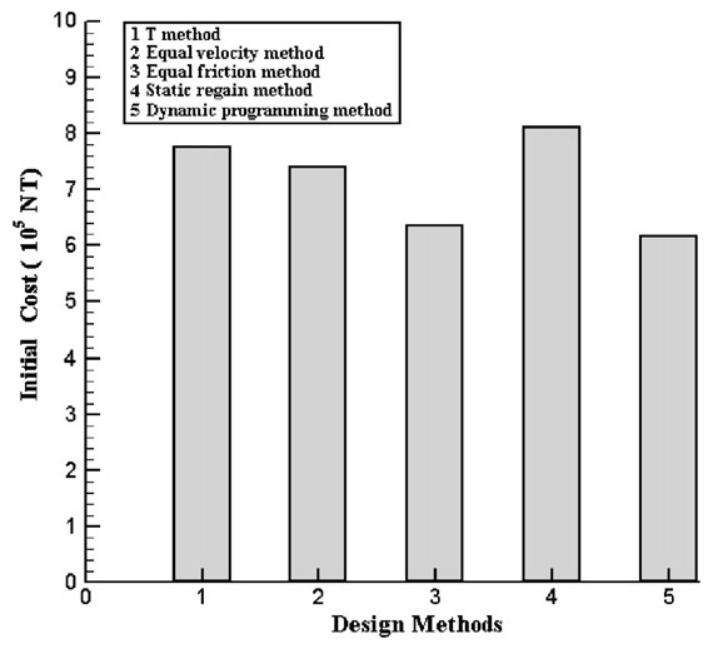

Fig. 7. Comparison of initial cost.

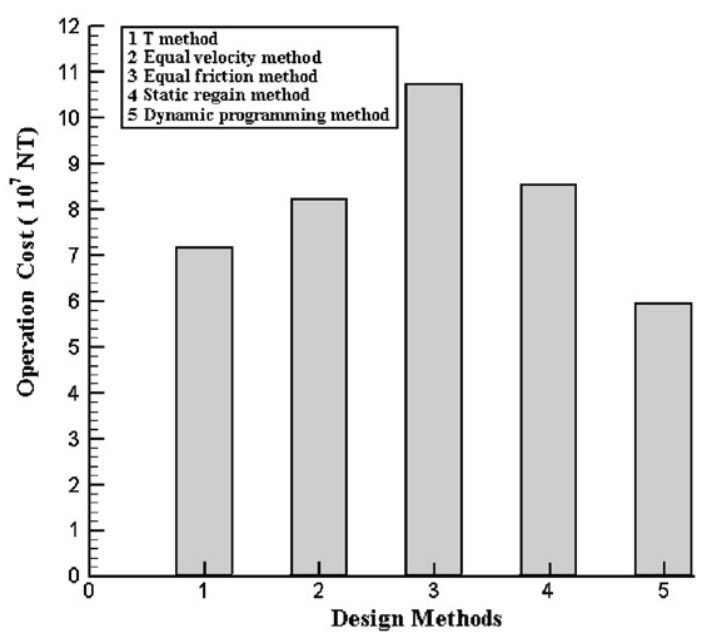

Fig. 8. Comparison of operating cost.

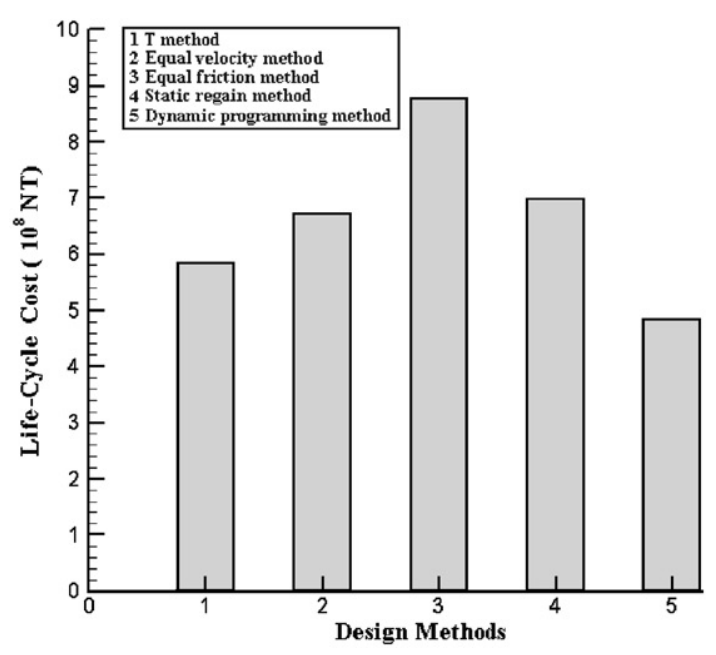

Fig. 9. Comparison of life-cycle cost. 
results attained fall within the range of duct diameter and flow velocity. Although the T-method can also design a duct system that achieves the lowest life-cycle, its basis of optimization is established on the selection of the optimal initial cost and energy cost. The control of duct diameter and flow velocity may be sacrificed as a result.

\section{References}

[1] ASHRAE Fundamentals Handbook, Atlanta: ASHRAE, 1997 [Chapter 32].
[2] Tsal RJ, Behls HF, Mangel R. T-method duct design, Part I: optimization theory. ASHRAE Transactions 1988;94(2):90-111.

[3] Tsal RJ, Behls HF, Mangel R. T-method duct design, Part II: calculation procedure and economic analysis. ASHRAE Transactions 1988;94(2):112-50.

[4] Mathews EH, Claassen DT. Problems with T-method. Building and Environment 1998;33(4):173-9.

[5] Bellman R. Dynamic programming. Princeton, NJ: Princeton University Press, 1957.

[6] Hillier FS, Ieberman JL. Introduction to operations research, 3rd ed. San Francisco, CA: Holdenday Inc, 1990.

[7] Tsal RJ, Behls HF, Mangel R. T-method duct design, part III: simulation. ASHRAE Transactions 1990;96(2):3-31. 